\title{
Understanding Link Quality in 802. I I Mobile Ad Hoc Networks
}

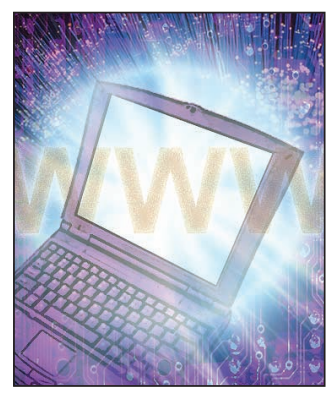

\author{
Gregor Gaertner and Vinny Cahill • Trinity College, Dublin
}

Mobile ad hoc wireless networks will extend the Internet into new territory, makingWeb services available "anytime, anywhere." This creates new markets in such areas as pervasive computing and traffic management.The authors show that the communication quality of current 802.I I ad hoc networks is low, and that users can experience strong fluctuations in link quality as a result. They identify key factors that cause these fluctuations and derive implications for application development. In particular, applications must tolerate frequent disconnections, network partitioning, and latency variations that are far more severe than in conventional networks.

W ireless local area networking is poised to become a mass-market technology. The past 10 years have brought a dramatic decline in WLAN technology prices of almost 40 percent per year. ${ }^{1}$ Analysts predict that the WLAN market will grow from 1.79 billion dollars in 2001 to 3.85 billion dollars in $2004,{ }^{2}$ and that 70 percent of all notebooks sold in 2004 will include $802.11{ }^{3}$ the dominant WLAN technology standard. ${ }^{2}$ The deployment of hotspot infrastructure for 802.11 networks is also increasing, giving people Internet access from public places such as airports, hotels, coffee shops, and companies. However, hotspot density is still far from covering whole cities or even city centers, forcing Internet application developers to confront two key questions:

- Despite the proliferation of WLAN-enabled devices, should developers wait for a more widely available infrastructure before exploiting ubiquitous Internet access?

- Should they leave untapped the potential of the many deployed 802.11-enabled devices to create new markets?

Fortunately, the answer in both cases is no. 802.11 supports both infrastructure and ad hoc operating modes. In infrastructure mode, nodes communicate using an intermediate base station. In ad hoc mode, nodes communicate directly with one another. To support communication between nodes that are not within range of each other, intermediate nodes act as message routers on behalf of their peers, building an infrastructure-independent, multihop store-and-forward network. This mode is available even if there are no base stations within the nodes' range. The advantage of these so-called mobile ad hoc wireless networks (Manets) is that, because they don't need an infrastructure, they are quickly deployable and highly flexible. Hence, Manets can extend the Internet beyond its traditional scope to new, previously inaccessible areas, making Web services available "anytime, anywhere." This will enable novel applications that will combine with existing software and infrastructure, and exploit available Internet knowledge. Enhanced automobile routing based on current traffic conditions, for example, could be deployed in cars based on the temporary formation of ondemand networks (for a good example of an existing mobility management solution on the Internet, see www.vmzberlin.de).

However, despite the Manet research community's rapid progress in recent years, fundamental 
problems, including efficient routing to enable reliable communication with highly mobile nodes and guaranteed quality of service (QoS), remain. The dynamic network's variable communication quality is significantly lower than that of conventional networks, and techniques and abstractions that hide this fact from application developers are still in the early stages. Real-world evaluations of such techniques, especially in urban environments, are also in their infancy. It is thus crucial that current application developers understand how link quality behaves in 802.11 ad hoc networks. Here, we present the results of our study, which examined factors that influence link quality and can help guide developers in deriving requirements for Manet applications.

\section{Mobile Ad Hoc Wireless Networks}

A Manet consists of nodes that create a dynamic, time-varying network without any infra-

\section{We identified shadowing by people and cars, the make of the wireless card, and the node height as significantly influencing link quality.}

structure or administrative support. Because nodes communicate only through broadcast over a limited transmission range, multihop communication is generally required to move messages through the network. Thus, intermediary nodes act as routers that forward received packets, establishing a path between the packet's source and destination.

\section{Current Challenges}

Because nodes move in and out of each other's range, the network topology changes frequently. Such changes must be communicated across the network to update routes accordingly. To maximize wireless communication channels' limited payload-transmission bandwidth, communications about topology changes must be minimized.

The network's dynamic nature, combined with adverse signal-propagation effects, raises issues that are difficult to address. In the physical layer, we need techniques that adapt to rapidly chang- ing channel characteristics to make link quality less sensitive to adverse signal-propagation effects. Because neighboring nodes share the same wireless medium and there is no central coordination, a distributed access control protocol must manage medium access in the link layer. Also, nodes that cannot communicate directly can still interfere and cause transmission errors at other nodes. Among other factors, this "hidden terminal problem" (see Ritter's work ${ }^{1}$ for one example) and imprecise network-state knowledge make medium access control especially challenging for multihop Manets. ${ }^{4}$

In the network layer, frequent topology changes and limited bandwidth place significant requirements on routing protocols. These protocols must balance the trade-off between

- high communication overhead (high bandwidth usage, low latency), which is required to propagate topology updates quickly, and

- low communication overhead (low bandwidth usage, high latency), which slows update propagation.

The optimal balance for different mobility dynamics is still subject to research. However, researchers have found an elegant solution that reduces latency: While conventional routing protocols initiate route discovery only after a path breaks, new proactive routing protocols predict future link states to initiate route discovery before a path breaks. Thus, a new path might be discovered before the old path fails. ${ }^{5}$

\section{Improving QoS}

With Manets, best effort is often the only QoS available. Specific guarantees - such as time bounds on packet delivery, available bandwidth, or probabilistic packet-loss guarantees - are not yet possible. Resource reservation seems to be key to realizing such guarantees. ${ }^{6}$ Because resources are severely limited, proactively predicting future system states can help reduce the unavoidable resource waste associated with providing QoS and thus make quality guarantees possible.

To improve protocol performance, we distilled three high-level concepts from the Manet literature. First, propagating topology updates only to directly or indirectly affected nodes saves valuable bandwidth and provides network stability. Second, using future network- and system-state predictions enables proactive decisions like packet rerouting 
and intelligent resource allocation before the network topology changes. Third, effectively coordinating node communication frees bandwidth for payload and reduces packet loss and traffic jams. However, all these concepts are as yet unable to overcome the low communication quality experienced in real-world Manets.

\section{Link Quality in 802.I I Networks}

Currently, the most promising 802.11 Manet applications are based in urban outdoor environments. Because 802.11 network deployments are relatively new in these environments, real-world experience with them is rare. We therefore ran an extensive series of outdoor experiments to assess 802.11 link quality, examining twelve factors that potentially influence it. To achieve practical results, we used standard, off-the-shelf hardware. Our results identified that five factors were highly influential:

- users shadowing (blocking) node links due to their own body orientation,

- other people shadowing node links,

- cars shadowing node links,

- the wireless card model, and

- node height.

Two factors - node orientation (associated shadowing by users aside) and the type of ground surface - had minimal influence on link quality. Five factors that we suspected would be influential but which proved to have no effect were small-scale movements, large-scale movements at different speeds, message length, payload pattern, and communication load without collisions.

\section{The Test Environment}

We conducted our experiments on the beach of a deserted island, where the benign environment let us individually assess influential factors without "noise" from multipath effects. (As we discuss later, the multipath effect occurs when a node receives waves from a single signal over multiple paths due to signal interference.) We conducted all experiments using two nodes that periodically sent messages to each other directly over the medium access control layer with a 2Mbit-per-second transmission rate and maximum transmission power. We did not use higher-level protocols because separating their protocol behavior from other influencing factors would have been awkward.
For each received packet, we recorded the link quality and used it to assess possible influencing factors. To measure link quality, we used the signal strength at which the node received packets. The received signal strength decreases with growing relative distance between sender and receiver by approximately the power of four if no shadowing and multipath effects occur. If a transmission's signal strength falls below a threshold, a packet is lost due to a transmission error. Signal strength responds quickly to changes in link quality, and current 802.11 cards use a large scale to describe it. Here, we show signal strength received at only one node because both communication directions were symmetrical (this is true only if you use the same wireless cards with the same transmission power, however). Missing signal strength values indicate that the corresponding packets were lost during transmission. To obtain the appropriate confidence level for our measurements, we conducted each experiment three times.

\section{Highly Influential Factors}

Two aspects are important for assessing the relevance of factors for influencing link quality: how strong the influence is and how likely it is to occur in practical networks. While the influence of some factors like shadowing by cars was expected, we were surprised by others, especially that users carrying a node frequently shadow links with their own body.

User orientation. In most wireless applications, users carry portable devices such as PDAs or notebooks. Because such nodes are rarely completely still and their antennas are often not strictly omni-directional, we assessed the influence on link quality of a node's orientation for all three axes in space. We found that link quality is affected only slightly, and the resulting minor link quality fluctuations can be ignored. However, a node's orientation is typically changed when the users holding the node changes the orientation of his or her own body. Thus, this user's body shadows the link if the body blocks the line of sight between the two communicating nodes. Figure 1 (next page) illustrates this effect. We placed two nodes at a fixed distance facing each other, while a person holding the node turned around. When the turning user faced away from the other node (around 180 degrees), the user's body reduced the signal strength to such an extent that packets 


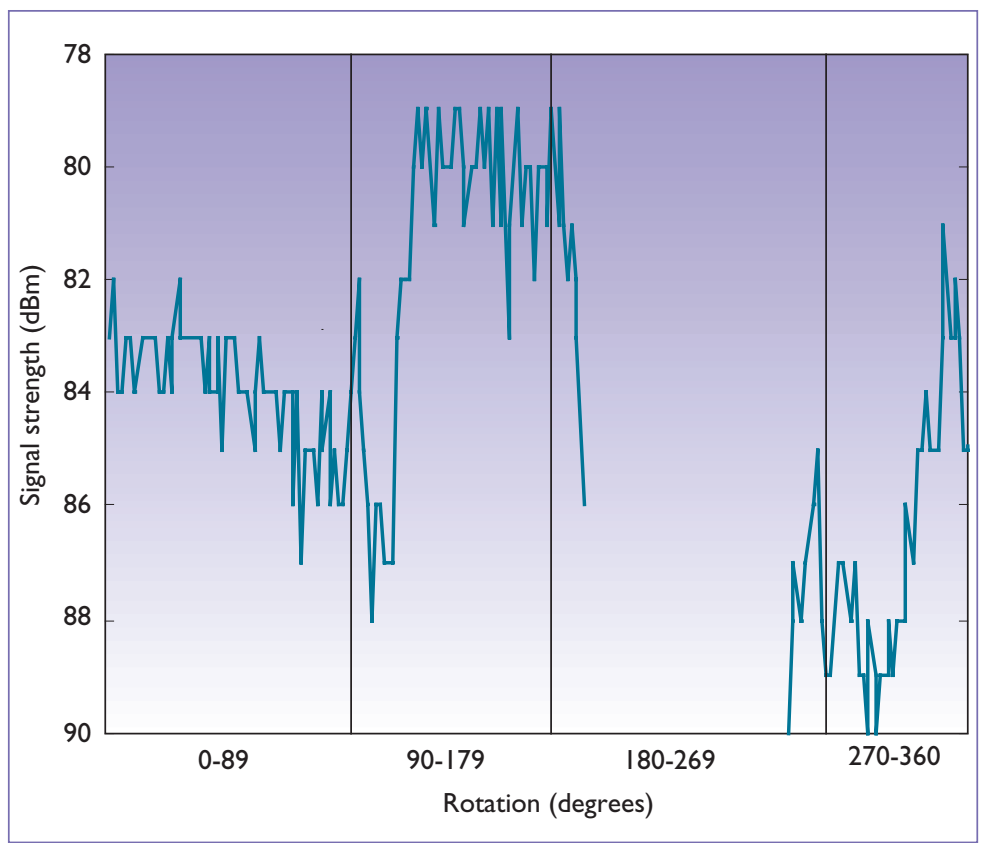

Figure I. Effect of a user shadowing a link while changing orientation. At I 80 degrees, signal strength was reduced to such an extent that packets were lost due to the user's body shadowing the link.

were lost. In further experiments, we found that the user's shadowing reduced the transmission range from 250 meters in open space to approximately 150 meters. Because people often change their orientation, such frequent and significant link quality fluctuations are likely to occur.

Dynamic object shadowing. In urban environments, dynamic objects such as people and cars are common. Obviously, these objects can shadow links and influence link quality. However, the significance of such shadowing varies. It is still possible, for example, to listen to a radio even if people are gathered around it, reducing the received signal. In our experiments, we observed that the affect of people and cars on link quality depends on their distance from the nearest node. As Figure 2 shows, a single car between two nodes that are 100 meters apart is sufficient to cause packet loss if the car is 1 meter away from either node. Even at 5 meters, the car continues to exert considerable influence. We found similar results for people, although their effect is weaker. Placing a single person in front of a node reduced signal strength severely (corresponding to shadowing by a user's own body), and the influence was still considerable at a distance of 1 meter. Because cars and people appear frequently in urban environments - especially in groups - severe linkquality fluctuations are likely.
802. I I card models. By definition, 802.11 wireless cards comply with the same specification; thus, if their transmission power is equal, we would expect the same behavior. However, as Figure 3 illustrates, cards from different makers can perform differently. In this experiment, we recorded the received signal strength at different distances with either two 802.11 Brand A cards or two Brand B cards. The range, signal-strength decay, and packet-loss threshold differed in the experiments with the two types of cards. This is most likely caused by differences in radio frequency design and signal processing algorithms.

Node height. In our experiments, node height also significantly influenced link quality. If one node was below belt height, link quality degraded independently of the other node's height. Thus, the performance of nodes placed at ground level is particularly poor and designers should avoid such placements in sensor networks. In military and disaster-recovery applications, frequent node height changes can occur and thus cause strong link quality fluctuations. For most applications, however, node placement is less significant as people typically carry nodes at a sufficient height.

\section{Additional Issues}

We assessed other candidate factors as well, but found them to have little or no influence on link quality. For example, the type of ground surface (such as sand or grass) had only a slight influence. Moreover, small-scale node movements (as when nodes are carried by a person, for example) had no affect on link quality. Large-scale movement at different speeds (evaluated up to 50 kilometers per hour) was also irrelevant for link quality (the Doppler shift is relevant only at much higher speeds).

We also found no influence from communication patterns including message length, payload pattern (random content versus 0/1 alternating bits), and communication load (such as a low versus high number of transmitted messages per unit time). However, a high communication load can cause collisions and thus packet loss due to the hidden terminal problem because nodes incorrectly sense that the busy communication channel is idle. This is particularly noticeable in areas where many nodes are located close together.

While our study was set up to test individual 
factors, additional factors influence link quality in urban environments. Most notably, wireless ratio propagation produces multipath effects. Because static and dynamic objects reflect, scatter, and diffract the signal, the node receiving a single signal's transmissions might receive multiple waves that are shifted due to different path lengths. Transmission errors caused by this multipath propagation can degrade link quality, even when the changes in node position are small. Wireless cards try to compensate for multipath effects using equalizers that periodically determine the channel characteristics. This compensation might suffer from higher node speed if an equalizer is not fast enough to determine the channel characteristics of new positions. Multipath effects are highly dependent on specific areas such that an individual assessment is virtually impossible.

\section{Conclusions for Application Development}

We identified several factors as causing severe link quality fluctuations. Because no communication abstractions are available yet that mask these fluctuations, developers need to consider the resulting consequences themselves. The varying link quality causes frequent short- and long-term link failures, such that network partitioning is the rule rather than the exception. Also, bandwidth is limited - especially by varying link availability in multihop transmissions. Moreover, significant link quality fluctuations are likely to cause high network latency variations.

Because of their dynamic infrastructure, node mobility, and adverse signal propagation effects, Manets have much lower communication quality than conventional networks such as the wired Internet. The original TCP protocol's poor performance in 802.11 networks illustrates this fundamental difference, ${ }^{8}$ and researchers are currently working on TCP protocol adaptations that specifically target Manets.

Manets' low communication quality imposes several requirements on application development. Not only are link failures frequent, but links can also oscillate between being available and unavailable over long time spans - a behavior that is not seen in conventional infrastructure networks. Applications must tolerate this oscillation, and programmers must define when they consider a link to have failed. Moreover, applications should be able to progress, even when they encounter network partitions. In contrast, wired network appli-

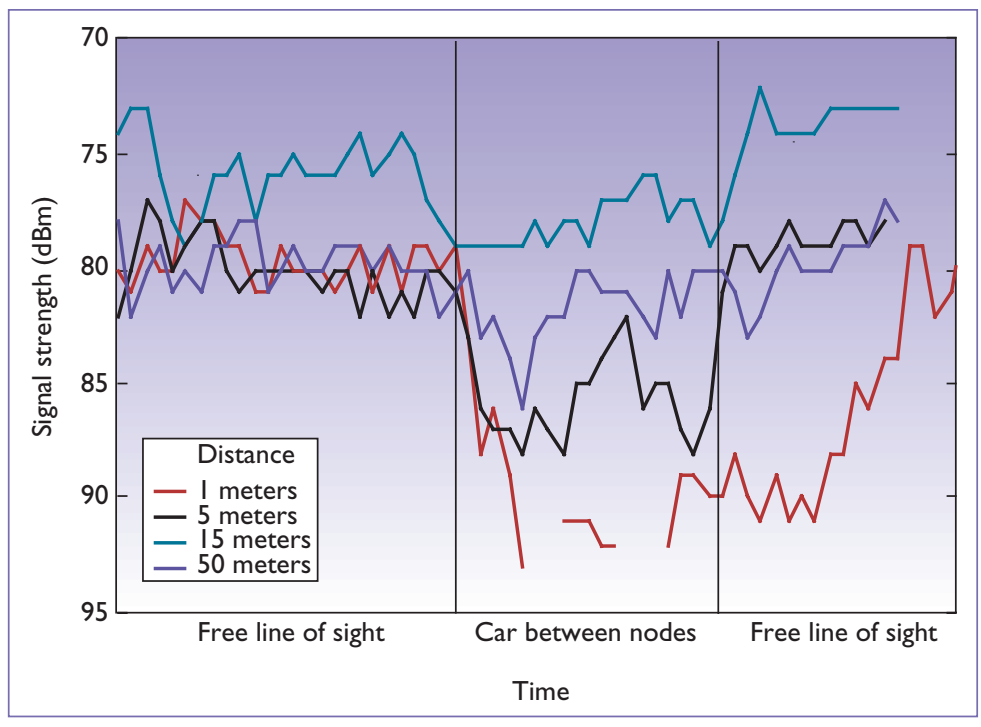

Figure 2. Effect of a car shadowing a link at different distances. At I meter away from either node, the car's influence was significant enough to cause packet loss. At 5 meters away, the car's influence was still considerable.

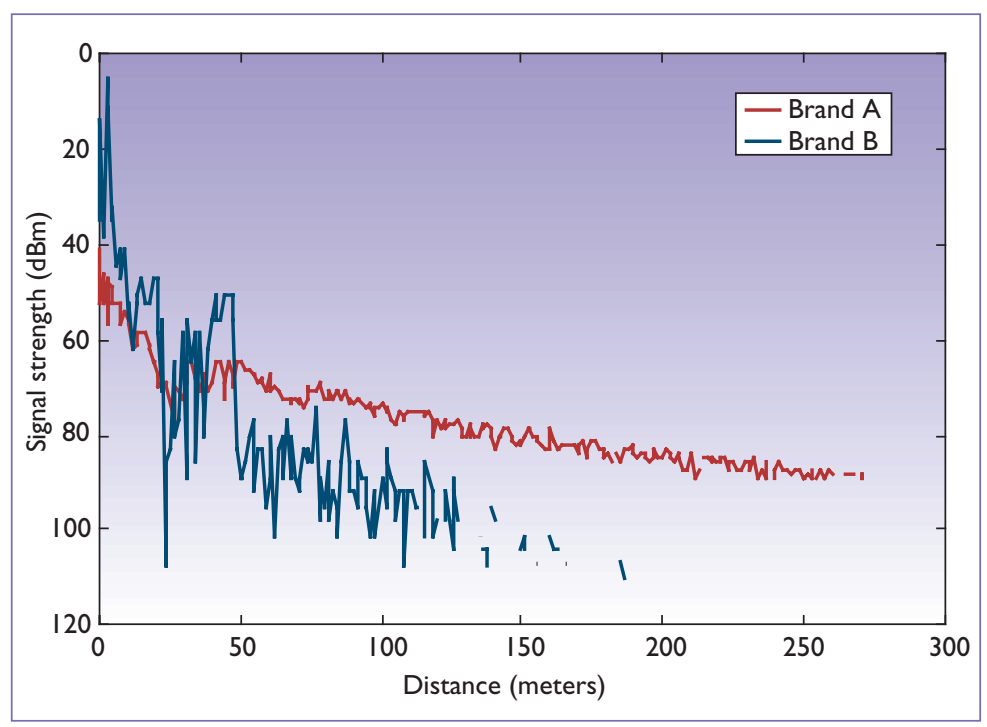

Figure 3.802. I I card behavior. The performance of different brands of card varied, despite 802. I I compliance and equivalent transmission power.

cations can treat partitioning as a special, shortlived phenomenon and block the progress of minority partitions.

Applications also need to efficiently store and garbage-collect state data to consistently merge network partitions when the topology allows. Furthermore, to reduce the communication volume and thus effectively use the available bandwidth, applications should preprocess the data at mobile 
nodes. An example is a sensor module that processes the measured data and distributes values only when measurements are outside a defined range. Finally, limited bandwidth combined with highly variable network latency make today's 802.11 Manets unsuitable for multimedia applications such as voice or video transmissions.

\section{Outlook}

Wide-scale deployment of 802.11 WLAN technology will allow the Internet to extend more deeply into our daily lives without the current temporal and spatial restrictions. This will create new markets and novel applications, particularly in the pervasive computing area. This prospect and 802.11's shortcomings are driving the development of several extensions to the original Wi-Fi standard. ${ }^{2}$ These physical- and link-layer extensions aim to provide a significantly higher communication quality in real-world urban environments, higher bandwidth, and guaranteed QoS. 802.11a and 802.11g, for example, provide much better compensation for multipath effects and a bandwidth of 54 Mbps. And 802.11e's prioritized medium access control and advanced polling techniques target guaranteed QoS that will enable voice and video transmissions. However, most extensions are still under discussion, and their wide-scale deployment is years away.

Novel network-layer algorithms will exploit 802.11 extensions and provide communication channels with higher reliability. Also, insights obtained from real-world Manet deployments will help provide robust solutions for application programming. In particular, we expect significant improvements in the areas of proactive, self-organizing routing protocols and QoS management, though the appropriate abstraction level for mobility and communication is still under discussion.

\section{Write for Spotlight}

potlight focuses on emerging technologies, or new aspects of existing technologies, that will provide the software platforms for Internet applications.

Spotlight articles describe technologies from the perspective of adeveloper of advanced Web-based applications.Articles should be 2,000 to 3,000 words. Guidelines are at www.computer.org/internet/dept.htm.

To check on a submission's relevance, please contact department editor Siobhán Clarke at siobhan.clarke@cs.tcd.ie.
Application developers who currently follow the Silicon Valley philosophy of "ready, fire, aim" will face widely fluctuating link quality in existing 802.11 Manets. To contend with the influences we've outlined here, applications must be designed robustly so that they can tolerate frequent longand short-lived disconnections and partitioning. Applications must also cope with low bandwidth, high latency fluctuations, and variable packet loss of communication channels - all of which occur much more frequently in Manets than in conventional infrastructure networks.

\section{References}

1. M.W. Ritter, "The Future of WLAN," ACM Queue, vol. 1, no. 3, 2003, pp. 18-27.

2. B. McFarland and M. Wong, "The Family Dynamic of 802.11," ACM Queue, vol. 1, no. 3, 2003, pp. 28-38.

3. ANSI/IEEE Standard 802.11, International Standard ISO/IEC 8802-11: 1999(E), IEEE, 1999.

4. S. Xu and T. Saadawi, "Does the IEEE 802.11 MAC Protocol Work Well in Multihop Wireless Ad Hoc Networks?" IEEE Comm., vol. 39, no. 6, 2001, pp. 130-137.

5. T. Goff et al., "Preemptive Routing in Ad Hoc Networks," Proc. 7th Int'l Conf. Mobile Computing and Networking (MOBICOM 2001), Springer Verlag, 2001, pp. 43-52.

6. S. Chakrabarti and A. Mishra, "QoS Issues in Ad Hoc Wireless Networks,” IEEE Comm., vol. 39, no. 2, 2001, pp. 142-148.

7. T.S. Rappaport, Wireless Communications - Principles and Practice, 2nd ed., Prentice Hall, 2002, pp. 120-125.

8. G. Holland and N. Vaidya, "Analysis of TCP Performance over Mobile Ad Hoc Networks," Wireless Networks, vol. 8, no. 2-3, 2002, pp. 275-288.

Gregor Gaertner is a PhD student in the Distributed Systems Group of the Department of Computer Science at Trinity College Dublin. His research interests include 802.11 Manets, link quality prediction, and group communication. He received a master's degree, with distinction, in computer science from Ilmenau Technical University, Germany. His thesis, A Replication Concept for a Distributed Discourse System on the Internet, was published as a book in the GMD Research Series. Contact him at gregor.gaertner@cs.tcd.ie.

Vinny Cahill is associate professor of computer science at Trinity College Dublin, where he leads the Distributed Systems Group. His research interests include middleware for mobile and context-aware computing. He received a $\mathrm{PhD}$ in computer science from the University of Dublin. He is a member of ACM and the IEEE Computer Society. Contact him at vinny.cahill@cs.tcd.ie. 